\title{
Laser-Based Propagation of Human iPS and ES Cells Generates Reproducible Cultures with Enhanced Differentiation Potential
}

\author{
Kristi A. Hohenstein Elliott, ${ }^{1}$ Cory Peterson, ${ }^{1}$ Anuradha Soundararajan, ${ }^{2}$ Natalia Kan, ${ }^{3}$ \\ Brandon Nelson, ${ }^{3}$ Sean Spiering, ${ }^{3}$ Mark Mercola, ${ }^{3}$ and Gary R. Bright ${ }^{2}$
}

${ }^{1}$ Intrexon Corporation, Cell Engineering Unit, 6620 Mesa Ridge Road, San Diego, CA 92121, USA

${ }^{2}$ Cyntellect, Inc., 6620 Mesa Ridge Road, San Diego, CA 92121, USA

${ }^{3}$ Sanford-Burnham Medical Research Institute, 10901 Torrey Pines Road, La Jolla, CA 92037, USA

Correspondence should be addressed to Kristi A. Hohenstein Elliott, khohenstein28@gmail.com

Received 15 September 2011; Revised 3 February 2012; Accepted 3 February 2012

Academic Editor: Mohan C. Vemuri

Copyright ( 92012 Kristi A. Hohenstein Elliott et al. This is an open access article distributed under the Creative Commons Attribution License, which permits unrestricted use, distribution, and reproduction in any medium, provided the original work is properly cited.

\begin{abstract}
Proper maintenance of stem cells is essential for successful utilization of ESCs/iPSCs as tools in developmental and drug discovery studies and in regenerative medicine. Standardization is critical for all future applications of stem cells and necessary to fully understand their potential. This study reports a novel approach for the efficient, consistent expansion of human ESCs and iPSCs using laser sectioning, instead of mechanical devices or enzymes, to divide cultures into defined size clumps for propagation. Lasermediated propagation maintained the pluripotency, quality, and genetic stability of ESCs/iPSCs and led to enhanced differentiation potential. This approach removes the variability associated with ESC/iPSC propagation, significantly reduces the expertise, labor, and time associated with manual passaging techniques and provides the basis for scalable delivery of standardized ESC/iPSC lines. Adoption of standardized protocols would allow researchers to understand the role of genetics, environment, and/or procedural effects on stem cells and would ensure reproducible production of stem cell cultures for use in clinical/therapeutic applications.
\end{abstract}

\section{Introduction}

Human embryonic and induced pluripotent stem cell (ESC, iPSC) lines have been derived and maintained in a variety of ways, creating extensive variability and inconsistency from laboratory to laboratory. Currently, ESC and iPSC lines are cultured under diverse conditions, involving numerous methods of expansion, both feeder-dependent and feederindependent matrices, and a variety of medium formulations [1-8]. Large-scale practical utilization of human ESCs and iPSCs for drug discovery applications, developmental and disease models, and regenerative therapeutic applications, will require more consistent and scalable culturing methods. Likewise, generation of GMP-quality stem cell lines will require standardized, traceable methods for stem cell derivation and expansion [9].

Manual passage, using specialized stem cell knives, razors, or pipettes to physically section stem cell colonies, is widely accepted as the best method for propagation of human ESC and iPSC lines. Manual propagation of stem cell lines does not involve the use of enzymes and therefore is thought to better maintain genetic stability of human ESCs and iPSCs in long-term culture [10-14]. Other benefits of manual expansion of stem cell cultures include passage of similar sized cell clumps, low cellular trauma, and selective transfer of specific undifferentiated colonies [7, 15]. However, scale-up of multiple stem cell lines using these methods is unattractive because of the high labor cost, inconsistency of output associated with varying expertise, risk of contamination, and the inability to effectively automate. Due to these technical demands associated with manual passage, routine propagation of most human ESC and iPSC lines is often performed using enzymatic passage $[5,6]$. Enzymatic methods (e.g., using accutase, collagenase, dispase, trypsin, or TrypLE) are usually used for large-scale expansion and are well suited for automated platforms [16]. However, these methods are highly problematic as enzymatic dissociation results in variable-sized colonies leading to significant 
inconsistency among cultures [7]. Also, human ESCs/iPSCs do not survive well as single cells which limit the utility of enzymatic propagation [17]. While chemical compounds (e.g., ROCK inhibitor) may be used to promote survival of dissociated stem cells, these compounds do not alleviate the heterogeneity associated with enzymatically passaged human ESC and iPSC cultures, nor is the full impact of routine usage of such compounds known [18]. The large heterogeneity in colony size also limits the usefulness of high density plates, such as 96-well and 384-well plates, for higher throughput applications.

This study reports a novel approach for the expansion of human stem cell lines using laser-based propagation. Lasermediated passaging was performed by precise cutting of human stem cell cultures by a laser into specific sized cell sections. These cell sections were transferred by simple pipetting to new culture dishes for propagation. The cell sections were of uniform size leading to greater uniformity of the resulting colonies. Additionally, enzyme-free conditions were maintained throughout and all processing occurred within a sterile, closed environment. Operating within standard format multiwell plates enable incorporation of automated robotic systems for scalable delivery of standardized stem cell cultures.

Laser-mediated propagation maintained the quality and pluripotency of ESC/iPSCs and led to enhanced differentiation potential. This approach removes the variability associated with passaging stem cells, which should greatly improve the evaluation of gene expression signatures, genetic/epigenetic profiles, and differentiation capabilities/efficiencies of stem cell lines. Laser-mediated ESC/iPSC passage significantly reduces the expertise, labor, and time associated with manual passaging techniques and provides the basis for reproducible propagation of GMP-quality human stem cell lines.

\section{Materials and Methods}

2.1. Human ESC and iPSC Culture. Four human iPSC lines (BIMR 6, P15-40, and BIMR 14, P25-40 generated from adult fibroblasts, BIMR A, P20-50 and BIMR L, P30-45 generated from fetal fibroblasts, all transduced with retroviruses containing Oct4, Sox2, Klf4, and $c$-Myc genes, Burnham Institute for Medical Research (BIMR)) were cultured in KODMEM supplemented with $20 \%$ KnockOut Serum Replacement, 1\% GlutaMax, 1\% nonessential amino acids, $0.1 \mathrm{mM} 2$-mercaptoethanol, and $8 \mathrm{ng} / \mathrm{mL}$ bFGF (all from Invitrogen). iPSCs were expanded on PMEF-CFs (Millipore) or Matrigel (BD Biosciences, in medium conditioned using PMEF-CFs), medium was changed every day and cells were passaged at $1: 2-1: 8$ every 5-7 days. H9 human ESCs (P3565) were cultured using the same medium on PMEF-CFs and were passaged 1:2-1:4 every 5-7 days. Experiments involving H9 human ESCs were performed at the BIMR Stem Cell Core.

iPSCs were passaged by several methods including manual passage using a pipette tip or using StemPro EZPassage Disposable Stem Cell Passaging Tool (Invitrogen), enzymatic passage using collagenase IV (Invitrogen) or 0.05\% trypsin (Invitrogen), and laser-mediated passage using the LEAP Cell Processing Workstation (Intrexon Corp).

Stem cell colony size was determined by measuring the longest diameter of colonies from brightfield images and by manual counting of Hoechst (Invitrogen) stained nuclei from fluorescent images. Whole well brightfield images of stem cell cultures were acquired on LEAP (Intrexon Corp) and Celígo (Brooks Automation, Inc). To generate ESC/iPSC growth curves, $0.25 \%$ trypsin (Invitrogen) was used to produce a single-cell suspension of ESC/iPSCs which were then counted using a hemocytometer on days $0-5$ after passage.

Transfer efficiency after laser-mediated passage was determined by manual counting of the number of ESC/iPSC sections per well after sectioning (prior to section removal by pipetting), after removal of sections from the processed well, and after transfer of sections to new culture plates using whole well brightfield images. Two days after passage, passage efficiency was determined by manual counting of the number of alkaline phosphatase positive colonies in cultures containing transferred sections.

2.2. Laser-Mediated Passage. Optimal laser processing conditions were established assessing laser power, laser spot size, and density of laser spots for cutting stem cell colonies into sections with minimal loss of cells. Assessment was performed empirically by testing the ability of a given condition to consistently cut typical stem cell cultures across 96-, $12-$, and 6-well plates. Photothermal laser processing was chosen to minimize cell loss during processing [19]. Laser pulse powers from 3-10 $\mu \mathrm{J}$ and laser spot sizes from 10$25 \mathrm{um}$ were systematically evaluated for sectioning through stem cell cultures of varying thickness. It was determined that $\sim 8 \mu \mathrm{J}$ laser power delivered in a $10 \mathrm{um}$ spot size was sufficient to section cultures of all thicknesses. To create a continuous sectioning line, laser pulses were positioned $16 \mu \mathrm{m}$ apart in a line to effectively cut stem cell colonies. After processing, samples were washed, sections were dislodged by pipetting using normal iPSC/ESC medium, and all sections were transferred to fresh culture plates containing PMEF-CFs or Matrigel. Cells were passaged at $1: 2-1: 8$ every $5-7$ days.

2.3. Human ESC/iPSC Differentiation. Embryoid bodies (EBs) were generated using collagenase IV treatment of day 5 human iPSC cultures for $0.5-1.0$ hour to remove colonies from culture dishes. Colonies were grown in differentiation medium in suspension culture using Ultra Low Attachment plates (Corning). The longest diameter of resulting EBs was manually measured using brightfield images acquired on LEAP (Intrexon Corp) on day 4 or 5 of suspension culture.

Human iPSCs were induced to spontaneously differentiate in medium composed of KODMEM supplemented with 20\% KnockOut Serum Replacement, 1\% GlutaMax, 1\% nonessential amino acids, and $0.1 \mathrm{mM} 2$-mercaptoethanol (Invitrogen). EBs were grown in suspension culture for 8 days and then plated onto gelatin-coated plates and allowed to differentiate for an additional 8 days. Medium was 
changed every other day. Cultures were fixed on day 16 for immunocytochemical analyses.

Human iPSCs were induced to form cardiomyocytes by culturing EBs for 4 days in suspension culture in medium composed of KODMEM (Invitrogen) supplemented with $20 \%$ fetal bovine serum (FBS, Hyclone), $1 \%$ Glutamax, 1\% nonessential amino acids, and $0.1 \mathrm{nM} 2$-mercaptoethanol (Invitrogen). On the 4th day, EBs were plated onto gelatincoated plates and allowed to differentiate for an additional 18 days (22 total days). Medium was changed every other day. RNA was collected for QRT-PCR analyses on day 16, the number of contracting EBs was counted on days 16 and 22, and cultures were fixed for immunocytochemical analyses on day 22.

Human iPSCs were induced to form neural rosettes by culturing EBs for 7 days in suspension culture in medium composed of 50\% DMEM/F12, 50\% Neurobasal medium supplemented with glutamax, $0.5 \mathrm{x}$ N2 supplement, $0.5 \mathrm{x}$ B27 supplement (Invitrogen), $0.5 \mathrm{mM}$ ascorbic acid, $0.1 \%$ albumin, $4.5 \times 10^{-4}$ M MTG (Sigma), and bFGF ( $20 \mathrm{ng} / \mathrm{mL}$, Peprotech). On the 7th day, EBs were plated onto gelatincoated plates in the medium described above supplemented with EGF (20 ng/mL, Peprotech) and allowed to differentiate for an additional 4 days. On day 11, the number of EBs containing $\geq 1$ neural rosette was manually counted.

2.4. Immunocytochemistry. Cells were fixed in $4 \%$ paraformaldehyde in PBS for $15 \mathrm{~min}$, permeabilized in $0.1 \%$ TritonX100 in PBS for $5 \mathrm{~min}$, and then blocked for 1 hour in blocking buffer $(10 \%$ serum of the same species as the secondary antibody, $0.05 \%$ Triton X-100 in PBS). Cells were washed and incubated with primary antibodies in $1 \%$ serum (same species as the secondary antibody) in PBS for 2 hours at room temperature or overnight at $4^{\circ} \mathrm{C}$. Human iPSC/ESCs were characterized using the following antibodies: Oct4, Sox2, and Nanog (R \& D Systems), SSEA4 (Developmental Studies Hybridoma Bank), and TRA1-60 and TRA1-81 (Santa Cruz Biotechnology). Apoptosis was analyzed using the following antibodies: caspase-3 and cleaved PARP (BD Biosciences) with staurosporine treatment of iPSC cultures used as a control (10 $\mu \mathrm{M}$ staurosporine, 4 hour treatment). Differentiated iPSCs were characterized using the following antibodies: Nestin, Map2, ANP (NPPA), and Troponin I (Millipore), Brachyury and Sox17 (R \& D Systems), AFP and $\alpha$-actinin (Sigma), and $\alpha$-MHC (Developmental Studies Hybridoma Bank). Cells were then washed and incubated with Alexa Fluor-conjugated secondary antibodies (Invitrogen) for 2 hours. All antibodies were diluted according to manufacturer's instructions. Cell nuclei were stained with Hoechst (Invitrogen). All images were acquired using the LEAP system (Intrexon Corp).

2.5. Alkaline Phosphatase Staining. Cells were fixed in $4 \%$ paraformaldehyde in PBS for $15 \mathrm{~min}$ and stained with Fast Red TR hemi (zinc chloride) salt (Sigma) and Naphthol, ASMX phosphate alkaline solution (Sigma) in $\mathrm{H}_{2} \mathrm{O}$ for ${ }^{15}-$ $30 \mathrm{~min}$. All images were acquired using the LEAP system (Intrexon Corp).
2.6. Quantitative RT-PCR Analysis. RNA was prepared using the RNeasy Micro/Mini Kit (Qiagen) and cDNA synthesis was performed using the ABI High Capacity cDNA Reverse Transcription Kit. QRT-PCR was performed in triplicate for each primer set and in each cell sample using an $\mathrm{ABI}$ 7900 HT Sequence Detection System. Amplification was performed using the Taqman Univeral PCR Mastermix (ABI). Specific primers and probes for stem-cell-associated genes, differentiation-associated genes, and cardiomyocyte genes were obtained from ABI. Stem-cell-associated genes included Pou5f1 (Hs00999632_g1), Sox2 (Hs01053049_s1), Nanog (Hs02387400_g1), Tert (Hs99999022_m1), Zfp42 (Hs00399279_m1), Dppa2 (Hs00414515_m1), and Esg1 (Hs00988349_g1). Cardiomyocte-associated genes included Nkx2.5 (Hs00231763_m1), TnnI3 (Hs00165957_m1), Actn1 (Hs00998100_m1), Mef2C (Hs01554599_m1), Myh6 (Hs00411908_m1), and Nppa (Hs00383230_g1). Expression levels of all genes were normalized to Eukaryotic 18s rRNA (Hs99999901_s1), and then analyzed using the $2^{\Delta \Delta C t}$ method [20].

2.7. Karyotype Analysis. Live cell cultures were analyzed by Cell Line Genetics. Cytogenetic analysis was performed on twenty G-banded metaphase cells.

2.8. aCGH Analysis. Genomic DNA was collected and purified using the Gentra Puregene Cell Kit (Qiagen). Hybridization was conducted with the $44 \mathrm{~K}$ Human StemArray (Ambry Genetics), with a resolution of $\sim 24 \mathrm{~kb}$ over the entire genome and high resolution exonic coverage in known stemcell-associated genes, tumor suppressors, and oncogenes. Samples were hybridized to a sex-matched pooled normal reference DNA (Promega). Data was analyzed by Ambry Genetics using DNA Analytics (Agilent) and reported using genome build HG18.

2.9. Statistical Analysis. Statistical analyses were performed using GraphPad Prism with a $P$ value of $\leq 0.05$ considered to be significant. One way analysis of variance (ANOVA) with Bartlett's test for equal variances was performed to evaluate resulting colony sizes generated after passage by five techniques $(n=20$ colonies/sample, Figure 2(c)) and resulting EB sizes generated from laser-mediated, collagenase, and trypsin-passaged cells $(n=30 \mathrm{~EB} / \mathrm{sample}$, Figure 5(b)). Statistical analysis of QRT-PCR data $(n=3$, Figures $4(\mathrm{c})$ and $5(\mathrm{~d}))$ was performed using a two-tailed $t$-test.

\section{Results}

3.1. Optimization of Laser-Mediated Passage. Laser-mediated passaging conditions were optimized using four human iPSC lines and one human ESC line. Human iPSC/ESC cultures were initially passaged by standard methodology (i.e., collagenase treatment plus manual scraping of cultures) into plates containing mitomycin c-treated murine embryonic fibroblasts and cultured for 5 days in iPSC/ESC medium. Laser-mediated cutting of stem cell colonies into clumps or sections of cells was facilitated by addition of a reagent to 
increase photothermal absorption of the laser's energy by the culture medium [19]. Laser processing conditions were optimized with respect to laser power, laser spot size, and number of laser shots required to effectively cut cultures with minimal loss of cells.

To determine the impact of section size on resulting colony size, stem cell cultures were cut into square cell sections ranging from 75 to $300 \mu \mathrm{m}$ in size and transferred to new culture dishes by gentle pipetting (Figure 1(a), hiPSCs (top, middle), hESCs (bottom)). Sections below $75 \mu \mathrm{m}$ contained very few cells (<8 cells/section), whereas $300 \mu \mathrm{m}$ sections were too large to easily remove from the plate by gentle pipetting alone. Stem cell colony size and number of cells per colony were assessed by brightfield imaging and fluorescent staining of nuclei, respectively, after processing cultures into $100-250 \mu \mathrm{m}$ sections (Figure $1(\mathrm{~b})$ ). Human iPSC cultures sectioned into 100, 150, 200, and $250 \mu \mathrm{m}$ sizes resulted in sections containing $12,25,47$, and 68 cells, respectively (Figure 1(b), top). Three days after passage human iPSC colonies measured 306, 367, 493, and $693 \mu \mathrm{m}$ in diameter with $62,119,184$, and 283 cells per colony, respectively. Similar results were obtained with all iPSC cell lines (data not shown) and with human ESCs (Figure 1(b), bottom) using the same laser processing conditions.

For routine propagation of iPSC and ESC cultures, section sizes of $200 \mu \mathrm{m}$ were used, which allowed consistent splitting every 7 days. Notably, other groups have identified $200 \mu \mathrm{m}$ (50-100 cells) as the optimal clump size for passage $[1,5,15,21]$. Propagation of human iPSCs using $200 \mu \mathrm{m} \mathrm{sec-}$ tions was highly efficient with an average transfer efficiency of $91 \pm 2 \%$ with $93 \pm 5 \%$ of the transferred sections forming viable colonies for an overall passage efficiency of $85 \pm 3 \%$. Passage of human ESCs resulted in similar data (overall passage efficiency of $\sim 82 \%$, data not shown). Laser-mediated passage with the current system required a total of $\sim 50$ $90 \mathrm{~min}$ to process an entire plate of stem cells (depending on plate type using $200 \mu \mathrm{m}$ sections; with the majority of time $(>90 \%)$ spent for laser processing and only a few minutes spent by the user). iPSCs and ESCs cultured under feeder-free conditions were also successfully propagated using the same laser-mediated passage conditions. These data demonstrate that multiple stem cell lines can be propagated by laser-based passage and that the size of resulting colonies can be easily controlled by varying the input section size.

\subsection{Improved Consistency of Stem Cell Cultures. Laser-} mediated passage was compared with traditional passaging techniques, both manual and enzymatic. iPSC cultures (BIMR 6) were passaged by (1) laser-mediated passage using $200 \mu \mathrm{m}$ sections, (2) manual passage using the StemPro EZPassage Disposable Stem Cell Passaging Tool (Invitrogen), (3) manual passage using a pipette tip (performed by an individual with 6-years experience), (4) enzymatic passage using collagenase, and (5) enzymatic passage using trypsin. Manual passage approaches generated significantly more uniform colonies than the enzymatic methods. Comparing laser-mediated passage with collagenase-based passage, image analysis of laser-passaged cultures revealed more homogeneous colony formation than collagenase passaged cultures (Figures 2(a) and 2(b). Stem cell cultures passaged by all methods were analyzed with respect to colony diameter and cells per colony (Figure 2(c)). Laser-mediated passage resulted in the most uniform colonies measuring $240 \pm$ $43 \mu \mathrm{m}(18 \% \mathrm{CV})$ in diameter containing $45 \pm 7$ (16\% CV) cells per colony one day after passage. Enzymatic passage by collagenase or trypsin resulted in significantly variable sized colonies measuring $365 \pm 177 \mu \mathrm{m}(48 \% \mathrm{CV})$ and $172 \pm$ $97 \mu \mathrm{m}(56 \% \mathrm{CV})$ in diameter containing $90 \pm 42(47 \% \mathrm{CV})$ and $25 \pm 19(76 \% \mathrm{CV})$ cells per colony, respectively. Manual passage techniques using a pipette tip or the EZPassage tool resulted in more similar sized colonies measuring $214 \pm$ $9 \mu \mathrm{m}(37 \% \mathrm{CV})$ with $34 \pm 3(37 \% \mathrm{CV})$ cells per colony and $226 \pm 65 \mu \mathrm{m}(29 \% \mathrm{CV})$ in diameter with $37 \pm 9(25 \% \mathrm{CV})$ cells per colony, respectively. However, the EZPassage tool does not allow colonies growing at the edge of each well to be propagated, leaving $>25 \%$ of the culture unsectioned (data not shown). Statistical analysis of variance showed that the stem cell cultures propagated by laser-mediated passage varied significantly less than $(P$ value $<0.0001)$ cultures passaged manually or by enzymatic methods, demonstrating that laser-mediated passage results in more consistent stem cell cultures than all other methods. Comparable results were also obtained using the BIMR A iPSC line (data not shown).

\subsection{Pluripotency, Quality, and Stability of Stem Cells after} Laser-Mediated Passage. The effect of the laser on human iPSC and ESC quality and pluripotency was examined immediately following laser-mediated sectioning of stem cell cultures into $200 \mu \mathrm{m}$ sections. As shown in Figure 3(a), pluripotency markers such as Oct4, Sox2, Nanog, SSEA4, TRA1-60, and TRA1-81 were highly expressed in sectioned iPSC cultures. Image analysis demonstrated that all markers were expressed homogeneously across sections, even in cells right next to the laser sectioning lines. In addition, cells right next to the laser cutting lines did not show any significant increase in apoptosis, as measured by immunocytochemical analysis of activated caspase- 3 and cleaved PARP four hours after laser-mediated sectioning (Figure 3(b)). Incubation of replicate cultures overnight (i.e., cultures were sectioned into $200 \mu \mathrm{m}$ sizes and then given fresh medium) resulted in significant growth of cells into the areas previously sectioned using the laser. Morphological and immunocytochemical analysis of these cultures (using the same pluripotency and apoptosis markers above) indicated that cells regrown into the laser sectioning area were indeed undifferentiated human iPSCs. The laser was then used to section a wider area $(\sim$ $1000 \mu \mathrm{m}$ ) into cultures for analysis of growth over several days. Again, these cultures showed no change in morphology, apoptosis, and pluripotency marker expression, indicating that laser processing did not affect stem cell self-renewal or pluripotency (Supplemental Figure 1 of the supplementary material available online at doi:10.1155/2012/926463).

In addition, laser-mediated passage of human iPSCs and ESCs did not alter cell growth as the cells exhibited equivalent growth rates as compared with collagenase passaged cells after multiple rounds of expansion (Figure 3(c)). 

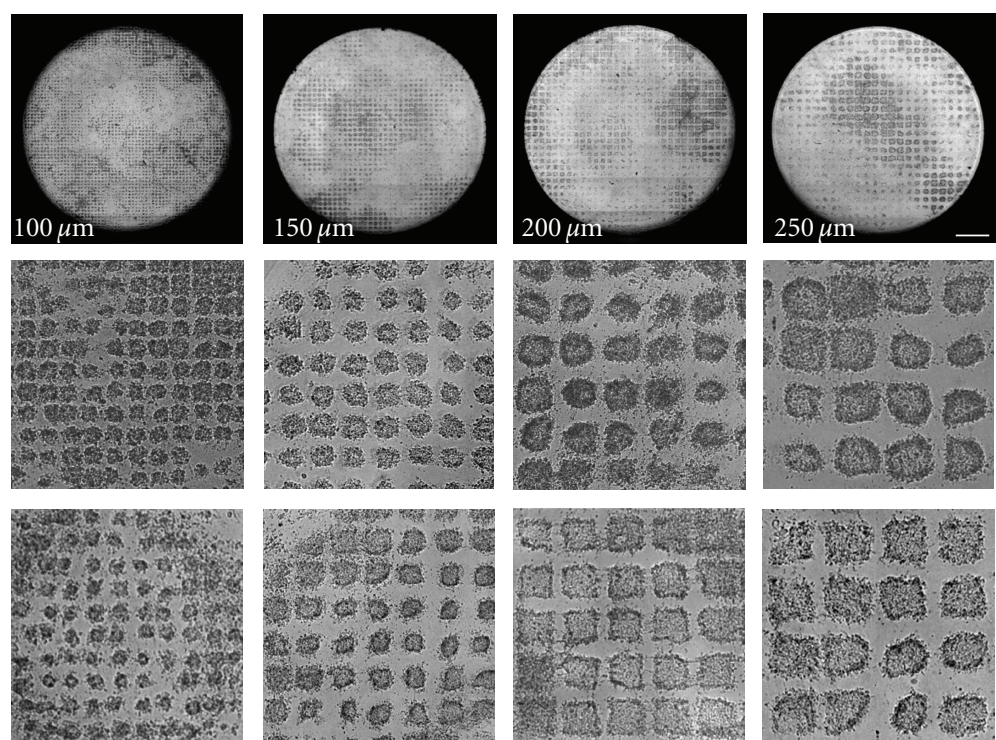

(a)
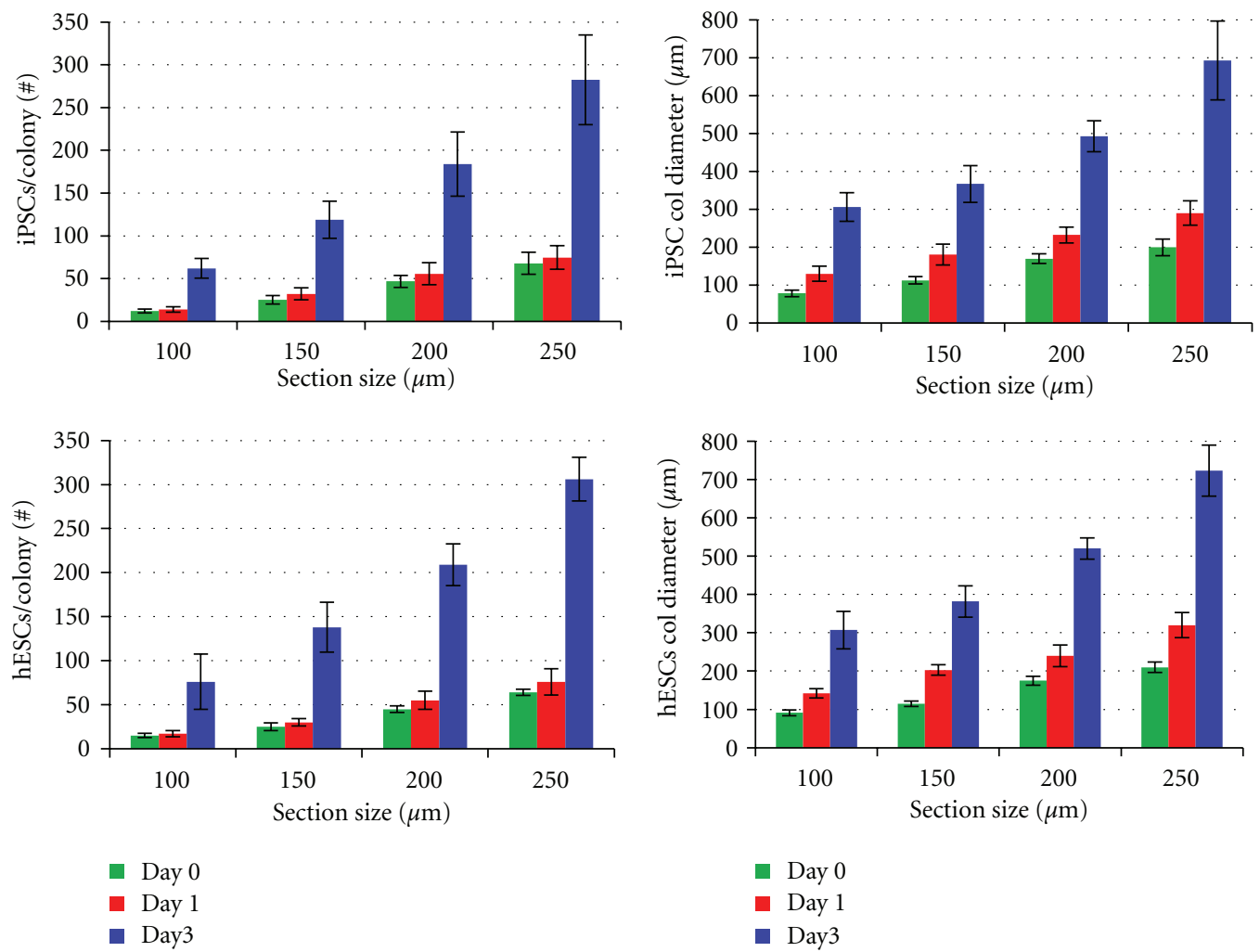

(b)

FIGURE 1: Stem cell colony size was controlled by section size using laser-mediated passage. (a) Brightfield images of human iPSC (top, middle) and ESC (bottom) cultures cut into 100-250 $\mu \mathrm{m}$ sections. Scale bar, $1 \mathrm{~mm}$. (b) Colony size over time following propagation of 100$250 \mu \mathrm{m}$ iPSC sections (top) or ESC sections (bottom) by laser-mediated passage. Number of cells per colony were manually counted using Hoechst stained cultures (left, $n=15$ colonies per data point). Longest diameter of each colony was manually measured using brightfield images (right, $n=15$ colonies per data point). Data are shown as mean \pm s.d.

To further assess the potential laser effects on human iPSC and ESC stability and pluripotency, stem cell cultures were propagated using laser-mediated passage over longterm culture (two iPSC lines were maintained for $>5$ passages
(5 weeks), one iPSC line was maintained for $>10$ passages (2.5 months), and BIMR 6 iPSCs and H9 ESCs were maintained for $>24$ passages ( $>6$ months)) and compared with replicate cultures passaged using collagenase. Image 

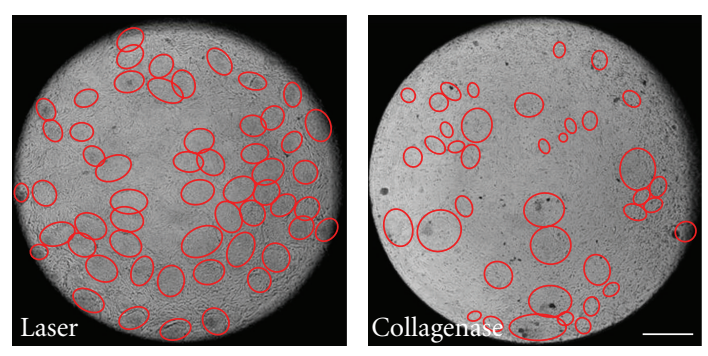

(a)

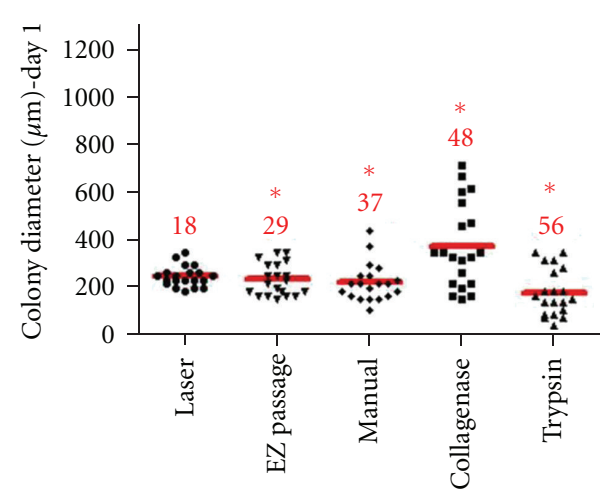

Passage method

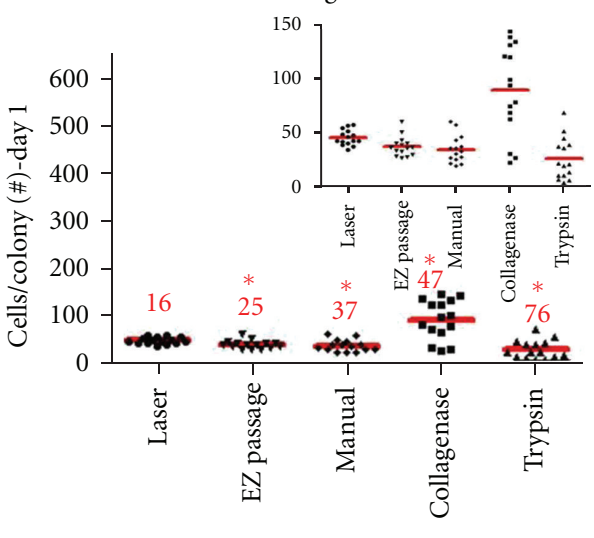

Passage method

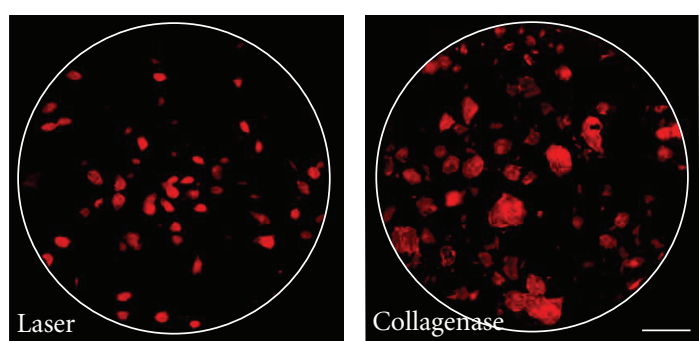

(b)

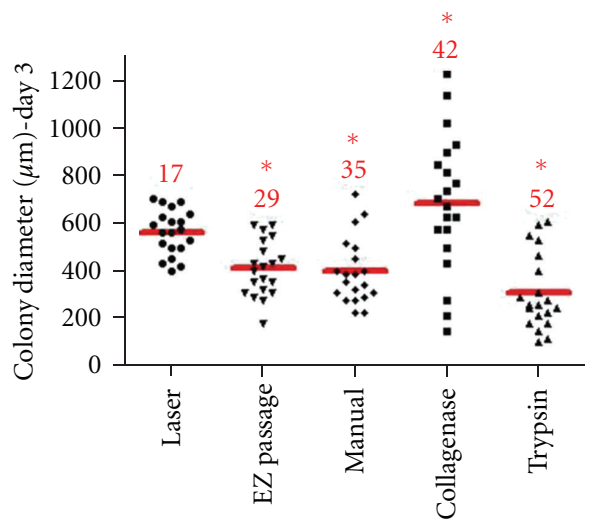

Passage method

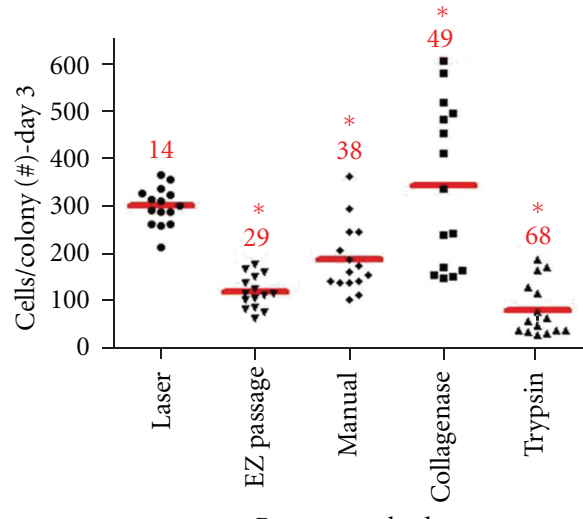

Passage method

(c)

FIGURE 2: Improved uniformity of stem cell cultures by laser-mediated passage. (a) Brightfield images of iPSC cultures 2 days after lasermediated passage ( $200 \mu \mathrm{m}$ sections, left) or collagenase passage (right). Colonies are shown by red outline. Scale bar, $1 \mathrm{~mm}$. (b) Alkaline phosphatase (AP) staining of iPSC colonies 1 day after laser-mediated passage ( $200 \mu \mathrm{m}$ sections, left) or collagenase passage (right). Scale bar, $1 \mathrm{~mm}$. (c) Colony size of iPSC cultures on days 1 and 3 after laser-mediated passage $(200 \mu \mathrm{m}$ sections), StemPro EZPassage Disposable Stem Cell Passaging Tool (EZ Passage), manual passage using a pipette tip, collagenase treatment, or trypsin dissociation of cells. Longest diameter of each colony was manually measured using brightfield images (top, $n=20$ colonies per data point). Number of cells per colony was manually counted using Hoechst stained cultures (bottom, $n=15$ colonies per data point). Data are shown as scatter plot with red line indicating mean. The CV is shown in red text above each sample. Asterisks $\left(^{*}\right)$ indicate variances that are statistically significant when compared to laser-mediated passage using ANOVA, with a $P \leq 0.05$ considered significant.

analyses of cultures propagated by laser-mediated passage showed no change in morphology with all cells exhibiting a high nuclear to cytoplasmic ratio typical of pluripotent stem cells (Figure 4(a)). Immunocytochemical analyses of these cultures demonstrated iPSCs and ESCs continued to express characteristic pluripotency markers including alkaline phosphatase (AP), Oct4, Sox2, Nanog, SSEA4, TRA1-60, and TRA1-81 (Figure 4(a)).
Visual comparison showed that human BIMR 6 iPSC cultures propagated using laser-mediated passage were of higher quality over time than those propagated using collagenase. Cultures propagated by laser-mediated passage contained many more undifferentiated, compact stem cell colonies with clear discernible colony borders compared to cultures propagated using collagenase. Collagenase passaged cultures had a greater tendency for spontaneous differentiation 

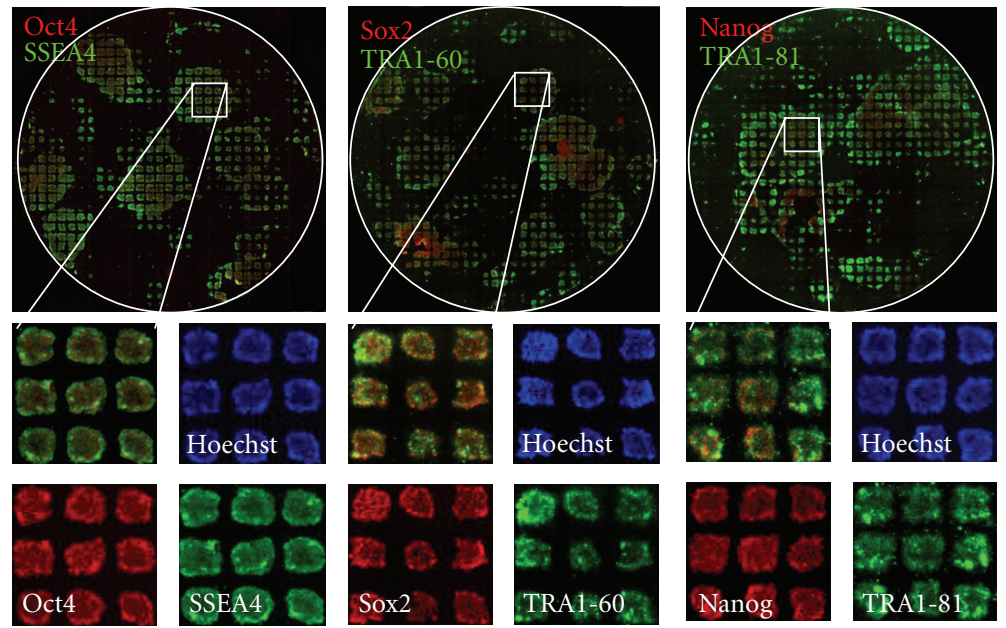

(a)
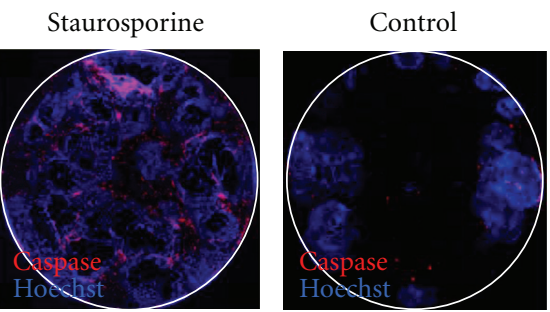

Sectioned iPSC culture
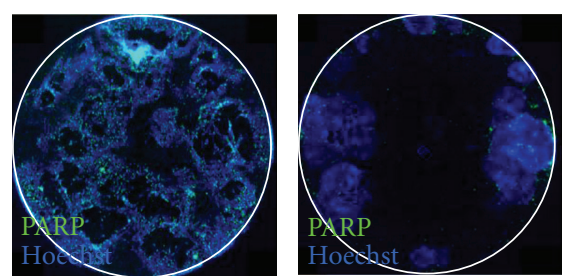

(b)
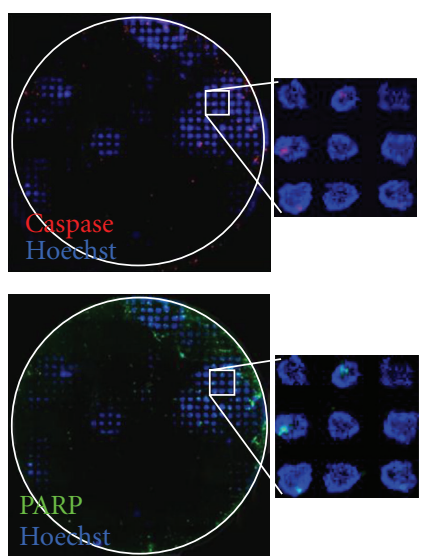

hESC growth after passage
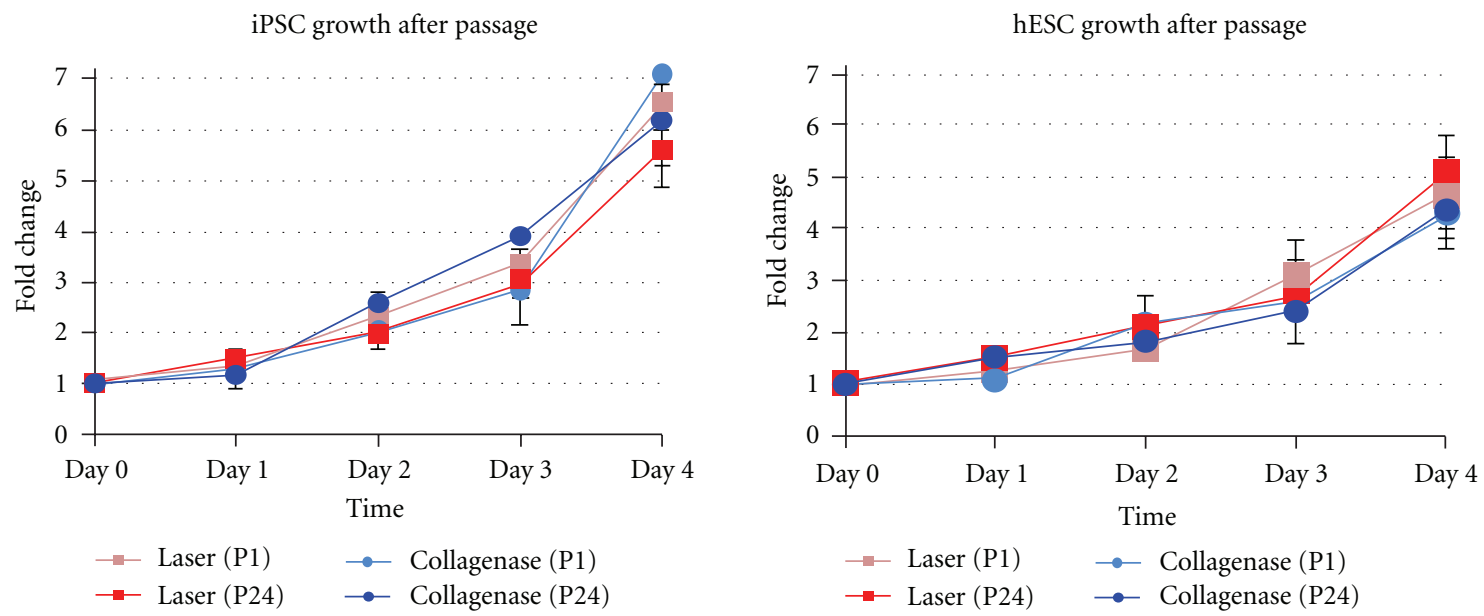

(c)

FIGURE 3: Quality of iPSC cultures after laser-mediated passage. (a) Immunocytochemical analysis of Oct4, Sox2, Nanog, SSEA4, TRA1-60, and TRA1-81 expression immediately following laser-mediated sectioning of iPSC cultures (BIMR L) into $200 \mu \mathrm{m}$ sections. Hoechst was used as a nuclear counterstain. Note that all markers are expressed homogeneously across iPSC clumps. Scale bar, $1 \mathrm{~mm}$. (b) Immunocytochemical analysis of apoptosis markers, caspase-3, and cleaved PARP, following laser-mediated sectioning of iPSC cultures. Hoechst was used as a nuclear counterstain. Scale bar, $1 \mathrm{~mm}$. (c) Analysis of iPSC (BIMR 6, left) and ESC (H9, right) growth following propagation using lasermediated passage or collagenase passage. $P$ indicates passage number. Data are shown as mean \pm s.d. $(n=3)$. 

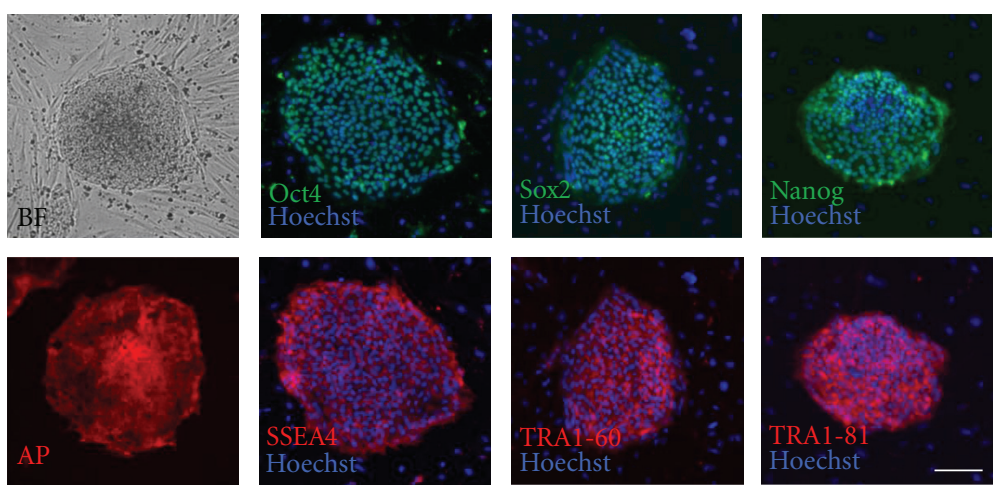

(a)

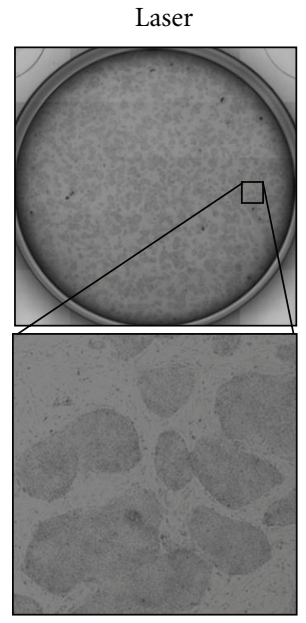

Collagenase

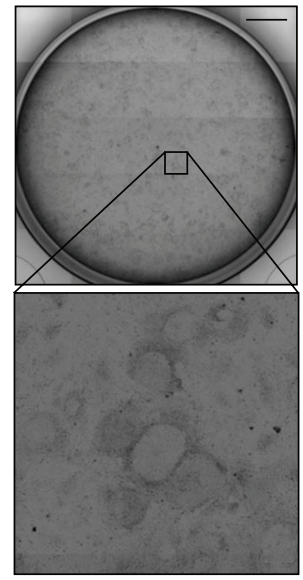

(b)

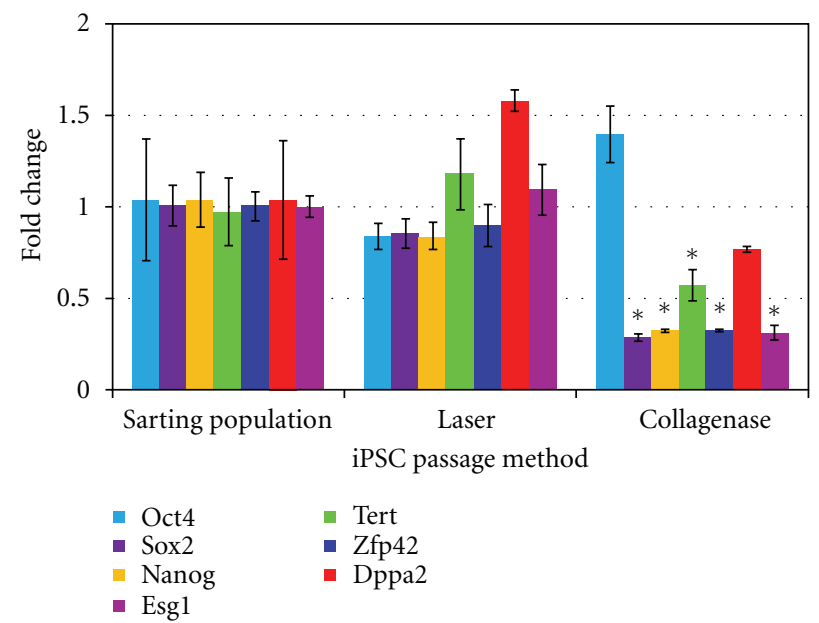

(c)

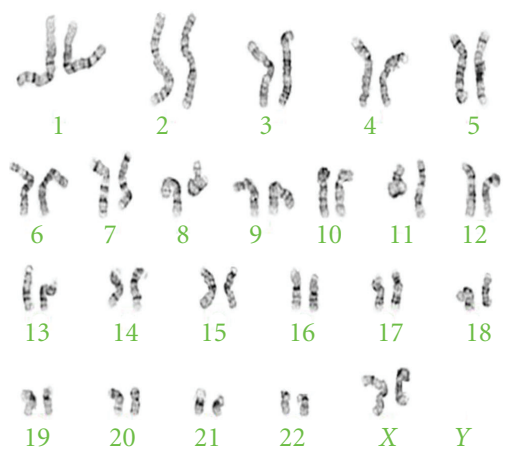

(d)

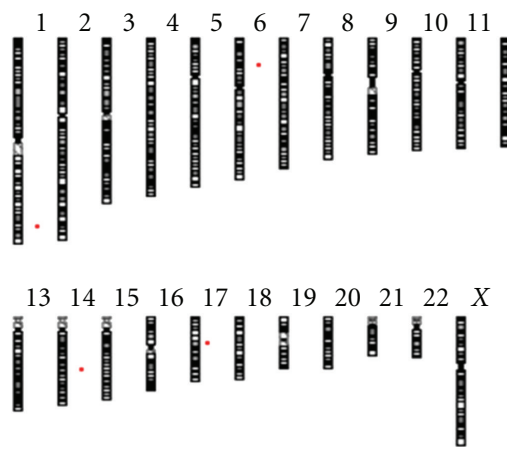

(e)

Figure 4: Pluripotency and stability of stem cells after laser-mediated passage. (a) Colony morphology (brightfield, BF) and immunocytochemical analysis of Oct4, Sox2, Nanog, alkaline phosphatase (AP), SSEA4, TRA1-60, and TRA1-81 expression in human ESCs (H9) after 24 consecutive laser-mediated passages. Hoechst was used as a nuclear counterstain. Scale bar, $250 \mu \mathrm{m}$. (b) Whole well brightfield images of human iPSC (BIMR 6) cultures after 10 consecutive laser-mediated passages or collagenase passages. Scale bar, 5 mm. (c) QRTPCR analysis of stem-cell-associated gene expression in iPSCs (BIMR 6) after 10 consecutive laser-mediated passages or collagenase passages. The asterisks $\left(^{*}\right)$ indicate values that are statistically significant compared with the starting population of iPSCs. The data are presented as mean \pm s.d. $(n=3)$. Statistical analysis was performed using $t$-test, with a $P$ value $\leq 0.05$ considered to be significant. (d) Normal karyotype of H9 human ESCs after 24 laser-mediated passages (6 months). (e) Schematic depicting genomic abnormalities of H9 ESCs at P35 (starting population) and at P59, after 24 laser-mediated passages (6 months), as determined by aCGH. No new subkaryotypic abnormalities were detected after 24 passages. Red bars indicate a deletion. Green bars indicate an amplification. See Table 1 for complete list of aberrations found in these cells. 
(Figure 4(b)). To quantify these phenotypic observations, QRT-PCR analyses were performed using known markers for undifferentiated stem cells. As shown in Figure 4(c), iPSCs propagated using laser-mediated passage continued to express high levels of stem cell-associated genes, including Oct4 (Pou5f1), Sox2, Nanog, Tert, Zfp42 (Rex1), Dppa2, and Esg1 (Dppa5), similar to the starting population of iPSCs (no statistical difference, $P$ value $\geq 0.1$ ). In contrast, iPSCs propagated using collagenase resulted in a significant decrease in expression of Sox2, Nanog, Tert, Zfp42, and Esg1 when compared to the starting population of iPSCs ( $P$ value $<0.05$, Figure 4(c)). Comparison of human ESC cultures passaged by both methods did not show any significant morphological changes or differences in gene expression (Supplemental Figure 2), although human ESC cultures were more established, later passage cultures than human iPSC cultures used in these experiments.

Data from multiple groups have shown that H9 human ESCs maintain a stable karyotype over long-term culture $[1,17,22]$. These cells were therefore used to examine the genetic stability of stem cells after long-term propagation using laser-mediated passage. Karyotype analysis of H9 ESCs after 24 consecutive laser-mediated passages showed no change in karyotype with all cells having a normal diploid karyotype (6 months, Figure 4(d)). To detect subkaryotypic alterations, array comparative genomic hybridization (aCGH) was also performed using the Stemarray (Figure 4(e), Table 1). No subkaryotypic alterations were detected in human ESCs propagated for 24 consecutive passages (P59) by laser-based passaging relative to the starting population $(\mathrm{H} 9, \mathrm{P} 35)$, suggesting that the genome of laser-mediated passaged cells is both normal and stable. It is important to note that both subkaryotypic and karyotypic alterations were observed in H9 ESCs after consecutive passaging by collagenase for 4 and 6 months, respectively (data not shown). In addition, no subkaryotypic changes were detected in human iPSCs (BIMR 6) propagated for 10 consecutive passages by laser-mediated passage relative to the starting population (data not shown). Although genetic stability of iPSCs was only analyzed after 2.5 months, taken together with 6-month human ESC results, these data suggest that the genome of laser-mediated passaged stem cells is and stable.

\subsection{Improved Differentiation Potential of EBs Generated after} Laser-Mediated Passage. To test the differentiation potential of these cells, in vitro differentiation assays of human iPSCs were performed after propagation using laser-mediated passage $(160 \mu \mathrm{m}$ sections) or enzymatic passage. iPSCs passaged by either methodology efficiently formed welldefined embryoid bodies in suspension culture which could differentiate into derivatives of all three primary germ layers including endodermal cells (Sox17, Afp), mesodermal cells/ cardiac muscle cells (brachyury, $\alpha$-MHC), and ectodermal cells/neurons (Nestin, Map2, Supplemental Figure 3). Morphological analysis of the resulting EB populations showed that EBs generated from laser-passaged iPSCs were more uniform in size than those generated from enzymatically passaged iPSCs (Figure 5(a)). To quantify these observations, the diameter of resulting EB populations was measured manually using images acquired on day 4 of suspension culture. As shown in Figure 5(b), laser-mediated passage resulted in significantly more uniform EBs $(374 \pm 56 \mu \mathrm{m}$; $15 \% \mathrm{CV}$ ) than enzymatic passage by either collagenase (336 $\pm 145 \mu \mathrm{m} ; 43 \% \mathrm{CV})$ or trypsin $(158 \pm 85 \mu \mathrm{m} ; 54 \% \mathrm{CV})$. Statistical analysis of variance showed that EBs generated using stem cell cultures propagated by laser-mediated passage were significantly more uniform $(P$ value $<0.0001)$ than EBs generated using enzymatically passaged cultures, demonstrating that laser-mediated passage results in more consistent EB cultures than other methods.

Several studies have shown that heterogeneity in human ESC colony size and resulting EB aggregate size results in variability in differentiation experiments and significant decreases in differentiation yields [23-26]. The effect of EB homogeneity on differentiation potential of human iPSCs into cardiomyocytes was examined. EBs were generated using iPSC colonies formed 5 days after laser-mediated passage $(160 \mu \mathrm{m}$ sections) or enzymatic passage. All EBs were differentiated using a standard multistage protocol, growing EBs in suspension culture for 4 days followed by adherent cell culture for an additional 18 days [27]. Cardiomyocyte differentiation potential was analyzed on day 22 of differentiation by manual counting of contracting EBs. EBs produced using enzymatically passaged iPSCs yielded a small proportion of beating EBs $(\sim 7 \%)$, whereas laser-mediated passaged iPSCs resulted in a significantly higher proportion $(\sim 60 \%)$ of contracting EBs (Figure 5(c)). QRT-PCR analyses confirmed these results with EBs generated from laser-mediated passaged cultures showing 3- to 51-fold higher expression of cardiomyocyte genes, Nkx2.5, Actn1 ( $\alpha$-actinin), Mef2C, Myh6 $(\alpha-M h c)$, TnnI3, and NppA (Anp), than EBs generated from collagenase passaged cultures (Figure 5(d)). Similarly, immunocytochemical analyses demonstrated that EBs produced from laser-mediated passaged iPSCs contained substantially more cardiac cells within each EB (i.e., EBs contained more cells staining positive for known cardiomyocyte markers) than EBs produced from collagenase passaged cells $(\alpha$-MHC and $\alpha$-actinin shown in Figure 5(e)). EBs generated from all populations stained positive for all markers tested including $\alpha$-MHC, $\alpha$-actinin, cardiac troponin, and NPPA (data not shown).

To further analyze the effect of EB homogeneity on differentiation potential, EBs generated from iPSC colonies formed 5 days after laser-mediated passage ( $160 \mu \mathrm{m}$ sections) or enzymatic passage were differentiated into neural rosettes using a modified multistep protocol. Ability to differentiate into neural rosettes was analyzed on day 11 of differentiation by manual counting of EBs containing $\geq 1$ neural rosette. EBs produced using laser-mediated passaged iPSCs resulted in 95\% of EBs containing neural rosettes, while EBs generated by trypsin or collagenase passaged iPSCs yielded only $29 \%$ and $32 \%$ of EBs containing neural rosettes, respectively (data not shown). These data indicate increased homogeneity in human iPSC colonies and resultant EBs result in significant increases in differentiation yield of iPSCs.

To investigate the effect of EB size on differentiation potential of human iPSCs were examined. iPSCs were 

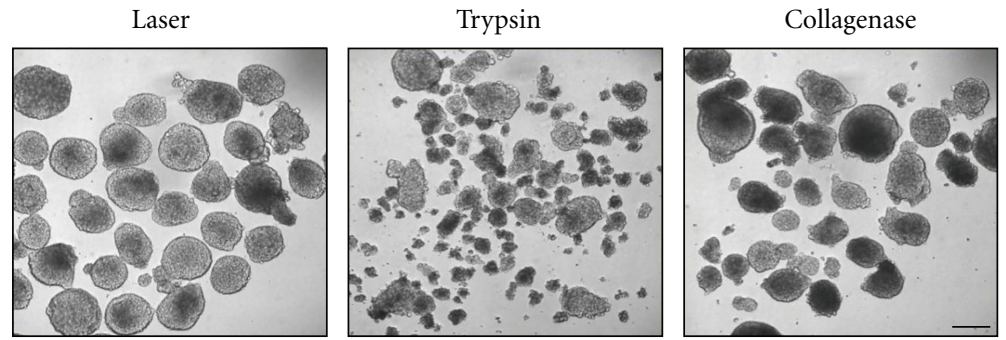

(a)

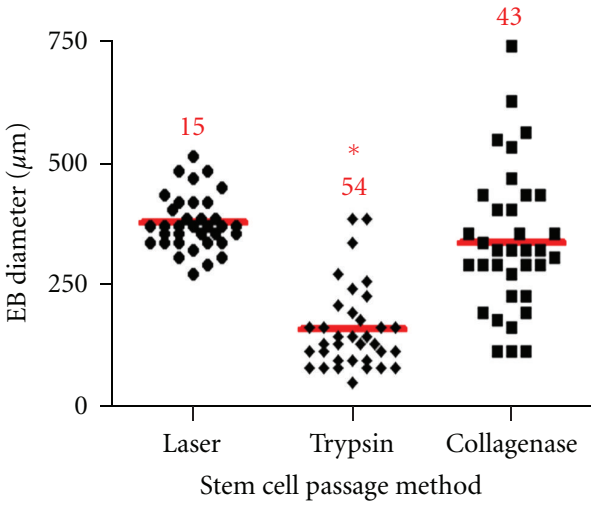

(b)

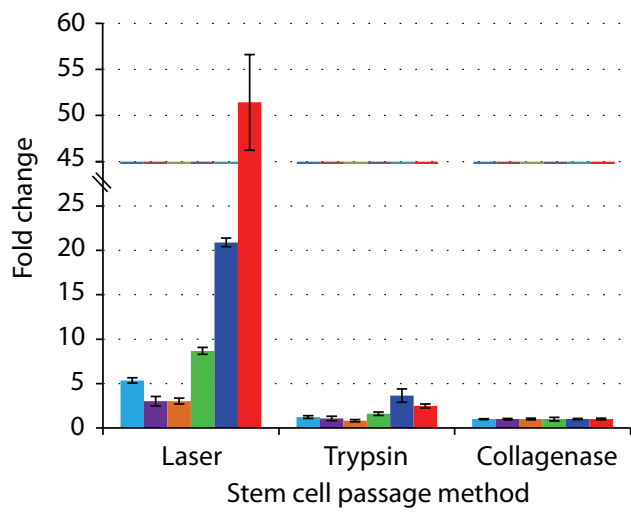

$\begin{array}{ll}\text { NKX2.5 } & \text { MYH6 } \\ \text { ACTN1 } & \text { TNNI3 } \\ \text { MEF2C } & \text { NPPA }\end{array}$

(d)

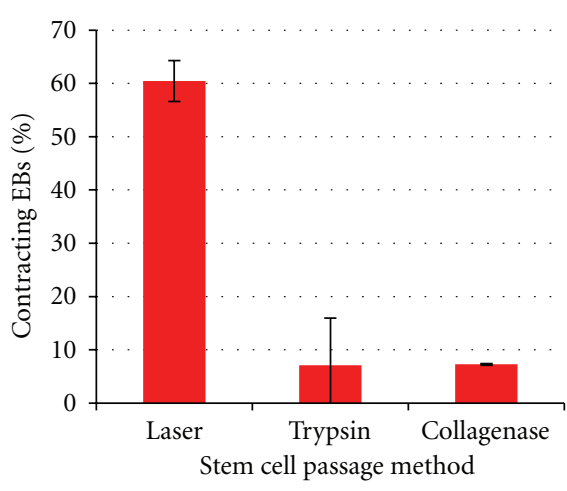

(c)
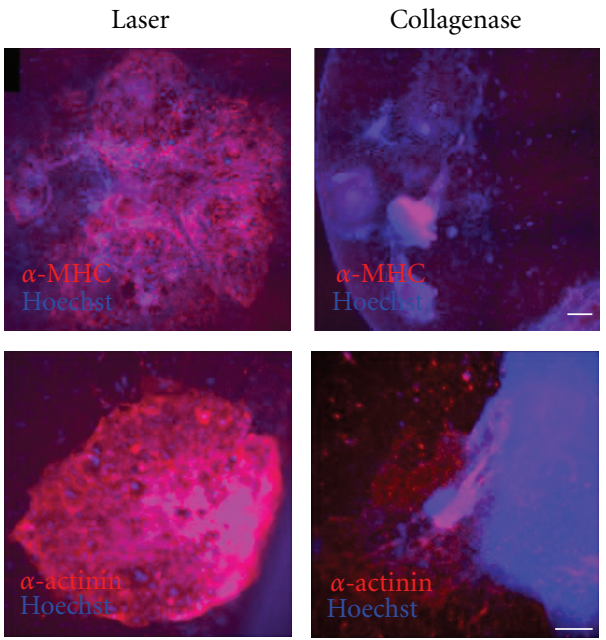

(e)

FIGURE 5: iPSCs propagated by laser-mediated passage differentiated more efficiently into cardiomyocytes. (a) Brightfield image of day 4 EBs generated from iPSC cultures (BIMR A) propagated by laser-mediated passage, trypsin dissociation, or collagenase treatment. Scale bar, $250 \mu \mathrm{m}$. (b) Size of EBs generated from iPSC cultures propagated by laser-mediated passage, trypsin dissociation, or collagenase treatment ( $n=35$ EBs per data point). Data are shown as scatter plot with red line indicating mean and CV shown in red text above each sample. The asterisks $\left(^{*}\right)$ indicate variances that are statistically significant when compared to laser using ANOVA, with $P \leq 0.05$ considered significant. (c) Percentage of EBs containing contracting areas. Data are shown as mean \pm s.d. $(n=2$ independent experiments containing 75 EBs/sample in each experiment). (d) QRT-PCR analysis of cardiomyocyte-associated gene expression in EBs generated using iPSC cultures propagated by laser-mediated passage, trypsin dissociation, or collagenase treatment. The asterisks $(*)$ indicate values that are statistically significant as compared with EBs generated from collagenase passaged iPSC cultures. The data are presented as mean \pm s.d. $(n=3)$. Statistical analysis was performed using $t$-test with $P \leq 0.05$ considered significant. (e) Expression of cardiomyocyte markers, $\alpha$-MHC and $\alpha$-actinin, in EBs generated from iPSC cultures propagated by laser-mediated passage or collagenase treatment on day 22 of cardiac differentiation. Hoechst was used as a nuclear counterstain. Scale bars, $250 \mu \mathrm{m}$. 
TABLE 1: Regions in H9 human ESCs with genomic abberations as determined by aCGH (corresponding schematic is shown in Figure 4(e)). No subkaryotypic alterations were detected in hESCs propagated for 24 consecutive passages (H9 P59) relative to the starting hESC population (H9 P35), suggesting that the genome of laser-mediated passaged cells is both normal and stable. Data is reported using genome built HG18. $\log _{2}$ ratios $\geq 0.6$ are amplifications (amp) or $\leq-1.0$ are deletions (del) found in all cells. $\log _{2}$ ratios $<0.6$ or $>-1.0$ represent mosaicism within the culture.

\begin{tabular}{lccccccc}
\hline Chromosome: region & Cytoband & $\begin{array}{c}\text { Size } \\
(\mathrm{Mb})\end{array}$ & \# Probes & Amp/Del & $\begin{array}{c}\text { H9 P35 } \\
\log _{2} \text { ratio }\end{array}$ & $\begin{array}{c}\text { H9 P59 } \\
\log _{2} \text { ratio }\end{array}$ & Annotations \\
\hline Chr1: 224141493-224195678 & $\mathrm{q} 42.12$ & 0.054 & 14 & Amp & 0.528 & 0.632 & LEFTY1, PYCR2, LEFTY2 \\
Chr6: 31663619-31691605 & $\mathrm{p} 21.33$ & 0.028 & 3 & Amp & 0.686 & 1.220 & LST1, NCR3, AIF1 \\
Chr12: $21580165-22105263$ & $\mathrm{q} 12.1$ & 0.282 & 12 & Del & -0.495 & -0.480 & GYS2, LDHB, KCNJ8, ABCC9, CMAS \\
Chr14: $62486603-62852257$ & $\mathrm{q} 23.2$ & 0.366 & 7 & Amp & 0.560 & 0.432 & KCNH5, RHOJ, GPHB5 \\
Chr17: $35097815-35153082$ & $\mathrm{q} 12$ & 0.055 & 15 & Amp & 0.469 & 0.485 & PERLD1, ERBB2, C17orf37, GRB7 \\
\hline
\end{tabular}

propagated by laser-mediated passage at varying section sizes (80, 160, and $240 \mu \mathrm{m}$ sections). Five days after passage, EBs were generated and differentiated into cardiomyocytes as described above. Homogeneous EB populations $(\leq 15 \%$ CVs) of varying sizes, 278,418 , and $528 \mu \mathrm{m}$ in diameter, were produced from 80,160 , and $240 \mu \mathrm{m}$ section sizes, respectively (Supplemental Figure 4). Analysis of cardiomyocyte differentiation potential showed that $55 \%$ of EBs generated from $160 \mu \mathrm{m}$ sections were contracting, while only $38 \%$ and $21 \%$ of EBs generated from $240 \mu \mathrm{m}$ and $80 \mu \mathrm{m}$ sections were contracting. Taken together, these data indicate that increased homogeneity in human iPSC colonies and resultant EBs, as well as EB size, significantly increase the differentiation yield of iPSCs. The ability to reproducibly generate uniform, size-specific colonies which subsequently result in more uniform, size-specific EB populations decreases variability in differentiation experiments and enhances differentiation yields of both ESC and iPSCs into specialized cell types.

\section{Discussion}

The lack of standardization in passage techniques for stem cell derivation and propagation is a major limitation within the stem cell field. Because universal protocols for human stem cell cultures have not been adopted, it is currently difficult to compare and interpret scientific data from cells cultured in different conditions. Passage method differences have significantly confounded the understanding of intraand interline differences in gene expression data, expression of stem cell- and lineage-associated markers, miRNA signatures, and epigenetic profiles [28-31]. Although human ESC lines have distinct genotypes, it is unlikely that reported differences in cell lines (e.g., up to 65\% variation in gene expression data across two ESC lines) can be attributed to genetic variation alone, as $<2 \%$ variation in gene expression has been found in adult human tissues of different individuals $[28,32,33]$. Likewise, discrepancies associated with differentiation protocols and reported differentiation capabilities and efficiencies of stem cells into specialized cell types may be due to the lack of standardization [34]. Adoption of standardized protocols should greatly improve determination of the role of inherent genetic variation, environmental niche, and/or procedural effects on stem cell quality, self-renewal, pluripotency, and differentiation potential.
Laser-mediated passage provides a novel method for expansion of human ESCs/iPSCs which can be used to create standardized, traceable procedures for the production of GMP-quality stem cell lines without requirement for enzymes. This method combines the benefits of both manual and enzymatic passage techniques, allowing efficient, automated passaging of undifferentiated stem cell cultures into uniform-sized stem cell sections within a sterile closed environment. Laser-mediated passage is compatible with a variety of culture methods including animal-free, feederfree-based conditions, and serum-free defined media conditions. Notably, this approach is not susceptible to interindividual variation reducing the need for skilled technicians to create high-quality stem cell cultures.

Laser-mediated passage does not involve the use of enzymes and therefore should better maintain the genetic stability of human ESCs and iPSCs in long-term culture (3-12 months, [10-14]). The results show that H9 ESCs maintained a stable karyotype over six months ( $>24$ passages). More importantly, laser-mediated passage did not induce subkaryotypic alterations over time in H9 ESCs (6 months, and iPSCs (2.5 months)) as monitored by aCGH. The more sensitive aCGH data suggests that laser-mediated passage maintains genetic integrity of human ESCs/iPSCs. Importantly, genetic abnormalities were detected in H9 ESCs after consecutive passaging by collagenase during the same time period. Results also showed that human iPSCs and ESCs propagated using laser-mediated passage maintained a normal stem cell morphology and continued to express high levels of stem-cell-associated genes and proteins. Although teratoma analyses were not performed on these cells, in vitro differentiation analyses of laser-mediated passaged iPSCs demonstrated the cells could spontaneously differentiate into derivatives of all three primary germ layers and could differentiate into cardiomyocytes and neural rosettes. In addition, iPSCs propagated by laser-mediated passage have been differentiated into motor neurons, RPE cells, endoderm progenitors, and hepatocytes-like cells (data not shown); taken together these data indicate that laser-mediated passage does not affect stem cell pluripotency. Likewise, lasermediated passage did not alter the growth rate of stem cells or increase expression of apoptotic markers, all supporting that the laser sectioning did not affect stem cell quality, selfrenewal, or pluripotency. 
Laser-mediated passage provides control of stem cell colony size. Regular passage schedules can be established by selection of section size. A section size of $\sim 200 \mu \mathrm{m}$ has enabled routine splitting of all ESC/iPSC lines every 7 days, allowing for more efficient planning of experiments. An overall passage efficiency of $85 \%$ combined with more uniform section sizes (20\% CV), enables a larger proportion of ESC/ iPSC colonies to contribute to culture expansion reducing the number of plates required for culture maintenance. Compatibility with conventional robotic systems enables scalability of culture needs. Additionally, the ability to control input section size, particularly smaller sizes, allows more effective creation of stem cell colonies in multi-well plates for largescale experimentation and screening purposes. It is also likely that stem cell section size will affect cryopreservation and genetic modification efficiency of ESCs and iPSCs [35-38].

Laser-mediated passage involves sectioning the entire well systematically without respect for the boundaries of the colonies. Well-established undifferentiated stem cell cultures are easily propagated using this technique. For newly derived ESC/iPSC cultures, early passage ESC/iPSC lines, or less stable lines, which tend to have more spontaneous differentiation, a combination of manual selection or laser purification of colonies followed by laser-mediated passage would be recommended. One of the more important results of this approach showed that over time, stem cell cultures (in particular, early passage iPSCs which tend to be more susceptible to differentiation than later passage, more established ESCs) are of higher quality than those maintained by collagenase treatment. It is likely that passage of homogeneous sections is important for maintaining undifferentiated stem cells and limiting the differentiation of colonies. Therefore, potentially early passage ESCs/iPSCs will require less colony isolation before expansion using laser-mediated passage.

One of the more important outcomes of this study showed that uniform human iPSC colonies produced after laser-mediated passage resulted in a more homogeneous population of EBs, with respect to size and shape, with greater differentiation efficiency as compared with typical EB cultures derived from enzyme passaged cultures. EBs generated from laser-mediated passaged iPSCs resulted in a significant increase in cardiomyocyte yield, with up to 8.5-fold greater beating incidence than EBs generated from collagenase passaged iPSCs. The ability to reproducibly generate uniform colonies using laser-mediated passage resulting in EBs that are more uniform in size and shape will decrease variability in differentiation experiments and enhance differentiation yields of both ESC and iPSCs into specialized cell types. These yield enhancements could significantly reduce the cost of stem cell experimentation both in terms of labor and materials. Potentially, uniform colony formation will also augment differentiation yields of stem cells when performing direct differentiation procedures (i.e., without an EB intermediate).

\section{Conclusions}

In conclusion, proper maintenance of human stem cells is essential for successful utilization of ESCs and iPSCs as tools in developmental and drug discovery studies and in regenerative medicine. Standardization is critical for all future applications of stem cells and necessary in order to fully understand the potential of these cells and the differences observed among varying stem cell lines and between ESCs and iPSCs. Laser-mediated passage is an innovative method for maintenance and expansion of stem cell lines, without introducing genetic instability, which is generically applicable to all cell lines and to all technicians regardless of skill. This approach provides an efficient, standardized protocol for the propagation of human ESCs and iPSCs, which should significantly reduce the inconsistency and variability within the stem cell field. Laser-mediated passage allows for traceability and ensures reproducible production of stem cell lines according to standard operating procedures, all of which are necessary to manufacture stem cells for use in clinical/therapeutic applications.

\section{Author's Contribution}

C. Peterson and A. Soundararajan contributed equally to the paper.

\section{Acknowledgments}

The authors would like to thank A. Elliott for assistance with aCGH experiments, and M. Koller, F. Kamme, and A. Pyle for suggestions and comments. The $\alpha$-MHC (developed by Fischman, D.A.) and SSEA4 (developed by Solter, D/Knowles, B.B.) antibodies were obtained from the Developmental Studies Hybridoma Bank developed under the auspices of the NICHD and maintained by The University of Iowa, Department of Biology, Iowa City, IA USA.

\section{References}

[1] J. A. Thomson, "Embryonic stem cell lines derived from human blastocysts," Science, vol. 282, no. 5391, pp. 1145-1147, 1998.

[2] C. Xu, M. S. Inokuma, J. Denham et al., "Feeder-free growth of undifferentiated human embryonic stem cells," Nature Biotechnology, vol. 19, no. 10, pp. 971-974, 2001.

[3] M. Richards, C. Y. Fong, W. K. Chan, P. C. Wong, and A. Bongso, "Human feeders support prolonged undifferentiated growth of human inner cell masses and embryonic stem cells," Nature Biotechnology, vol. 20, no. 9, pp. 933-936, 2002.

[4] M. Amit, C. Shariki, V. Margulets, and J. Itskovitz-Eldor, "Feeder layer- and serum-free culture of human embryonic stem cells," Biology of Reproduction, vol. 70, no. 3, pp. 837-845, 2004.

[5] B. E. Reubinoff, M. F. Pera, C. Y. Fong, A. Trounson, and A. Bongso, "Embryonic stem cell lines from human blastocysts: somatic differentiation in vitro," Nature Biotechnology, vol. 18, no. 4, pp. 399-404, 2000.

[6] C. A. Cowan, I. Klimanskaya, J. McMahon et al., "Derivation of embryonic stem-cell lines from human blastocysts," The New England Journal of Medicine, vol. 350, no. 13, pp. 13531356, 2004.

[7] K. O. Sun, S. K. Hee, B. P. Yong et al., "Methods for expansion of human embryonic stem cells," Stem Cells, vol. 23, no. 5, pp. 605-609, 2005. 
[8] L. M. Hoffman and M. K. Carpenter, "Characterization and culture of human embryonic stem cells," Nature Biotechnology, vol. 23, no. 6, pp. 699-708, 2005.

[9] C. Unger, H. Skottman, P. Blomberg, M. Sirac dilber, and O. Hovatta, "Good manufacturing practice and clinical-grade human embryonic stem cell lines," Human Molecular Genetics, vol. 17, no. 1, pp. R48-R53, 2008.

[10] J. J. Buzzard, N. M. Gough, J. M. Crook, and A. Colman, "Karyotype of human ES cells during extended culture," Nature Biotechnology, vol. 22, no. 4, pp. 381-382, 2004.

[11] M. M. Mitalipova, R. R. Rao, D. M. Hoyer et al., "Preserving the genetic integrity of human embryonic stem cells," Nature Biotechnology, vol. 23, no. 1, pp. 19-20, 2005.

[12] J. S. Draper, K. Smith, P. Gokhale et al., "Recurrent gain of chromosomes $17 \mathrm{q}$ and 12 in cultured human embryonic stem cells," Nature Biotechnology, vol. 22, no. 1, pp. 53-54, 2004.

[13] G. Caisander, H. Park, K. Frej et al., "Chromosomal integrity maintained in five human embryonic stem cell lines after prolonged in vitro culture," Chromosome Research, vol. 14, no. 2, pp. 131-137, 2006.

[14] S. N. Brimble, X. Zeng, D. A. Weiler et al., "Karyotypic stability, genotyping, differentiation, feeder-free maintenance, and gene expression sampling in three human embryonic stem cell lines derived prior to August 9, 2001," Stem Cells and Development, vol. 13, no. 6, pp. 585-597, 2004.

[15] A. Joannides, C. Fiore-Hériché, K. Westmore et al., "Automated mechanical passaging: a novel and efficient method for human embryonic stem cell expansion," Stem Cells, vol. 24, no. 2, pp. 230-235, 2006.

[16] S. Terstegge, I. Laufenberg, J. Pochert et al., "Automated maintenance of embryonic stem cell cultures," Biotechnology and Bioengineering, vol. 96, no. 1, pp. 195-201, 2007.

[17] M. Amit, M. K. Carpenter, M. S. Inokuma et al., "Clonally derived human embryonic stem cell lines maintain pluripotency and proliferative potential for prolonged periods of culture," Developmental Biology, vol. 227, no. 2, pp. 271-278, 2000.

[18] K. Watanabe, M. Ueno, D. Kamiya et al., "A ROCK inhibitor permits survival of dissociated human embryonic stem cells," Nature Biotechnology, vol. 25, no. 6, pp. 681-686, 2007.

[19] M. R. Koller, E. G. Hanania, J. Stevens et al., "High-throughput laser-mediated in situ cell purification with high purity and yield," Cytometry Part A, vol. 61, no. 2, pp. 153-161, 2004.

[20] K. J. Livak and T. D. Schmittgen, "Analysis of relative gene expression data using real-time quantitative PCR and the 2$\Delta \Delta$ CT method," Methods, vol. 25, no. 4, pp. 402-408, 2001.

[21] N. Heins, M. C. O. Englund, C. Sjöblom et al., "Derivation, characterization, and differentiation of human embryonic stem cells," Stem Cells, vol. 22, no. 3, pp. 367-376, 2004.

[22] E. S. Rosler, G. J. Fisk, X. Ares et al., "Long-term culture of human embryonic stem cells in feeder-free conditions," Developmental Dynamics, vol. 229, no. 2, pp. 259-274, 2004.

[23] E. S. Ng, R. P. Davis, L. Azzola, E. G. Stanley, and A. G. Elefanty, "Forced aggregation of defined numbers of human embryonic stem cells into embryoid bodies fosters robust, reproducible hematopoietic differentiation," Blood, vol. 106, no. 5, pp. 1601-1603, 2005.

[24] P. W. Burridge, D. Anderson, H. Priddle et al., "Improved human embryonic stem cell embryoid body homogeneity and cardiomyocyte differentiation from a novel V-96 plate aggregation system highlights interline variability," Stem Cells, vol. 25, no. 4, pp. 929-938, 2007.

[25] C. L. Bauwens, R. Peerani, S. Niebruegge et al., "Control of human embryonic stem cell colony and aggregate size heterogeneity influences differentiation trajectories," Stem Cells, vol. 26, no. 9, pp. 2300-2310, 2008.

[26] B. Valamehr, S. J. Jonas, J. Polleux et al., "Hydrophobic surfaces for enhanced differentiation of embryonic stem cell-derived embryoid bodies," Proceedings of the National Academy of Sciences of the United States of America, vol. 105, no. 38, pp. 14459-14464, 2008.

[27] C. Xu, S. Police, N. Rao, and M. K. Carpenter, "Characterization and enrichment of cardiomyocytes derived from human embryonic stem cells," Circulation Research, vol. 91, no. 6, pp. 501-508, 2002.

[28] C. Allegrucci and L. E. Young, "Differences between human embryonic stem cell lines," Human Reproduction Update, vol. 13, no. 2, pp. 103-120, 2007.

[29] C. Allegrucci, Y. Z. Wu, A. Thurston et al., "Restriction landmark genome scanning identifies culture-induced DNA methylation instability in the human embryonic stem cell epigenome," Human Molecular Genetics, vol. 16, no. 10, pp. 12531268, 2007.

[30] M. J. Abeyta, A. T. Clark, R. T. Rodriguez, M. S. Bodnar, R. A. Reijo Pera, and M. T. Firpo, "Unique gene expression signatures of independently-derived human embryonic stem cell lines," Human Molecular Genetics, vol. 13, no. 6, pp. 601608, 2004.

[31] H. Skottman, M. Mikkola, K. Lundin et al., "Gene expression signatures of seven individual human embryonic stem cell lines," Stem Cells, vol. 23, no. 9, pp. 1343-1356, 2005.

[32] X. Zeng, T. Miura, Y. Luo et al., "Properties of pluripotent human embryonic stem cells BG01 and BG02," Stem Cells, vol. 22, no. 3, pp. 292-312, 2004.

[33] L. L. Hsiao, F. Dangond, T. Yoshida et al., "A compendium of gene expression in normal human tissues," Physiol Genomics, vol. 7, no. 2, pp. 97-104, 2001.

[34] K. Osafune, L. Caron, M. Borowiak et al., "Marked differences in differentiation propensity among human embryonic stem cell lines," Nature Biotechnology, vol. 26, no. 3, pp. 313-315, 2008.

[35] X. Li, G. Meng, R. Krawetz, S. Liu, and D. E. Rancourt, "The ROCK inhibitor Y-27632 enhances the survival rate of human embryonic stem cells following cryopreservation," Stem Cells and Development, vol. 17, no. 6, pp. 1079-1085, 2008.

[36] R. Eiges, "Genetic manipulation of human embryonic stem cells by transfection," Methods in Molecular Biology, vol. 331, pp. 221-239, 2006.

[37] K. A. Hohenstein, A. D. Pyle, Y. I. C. Jing, L. F. Lock, and P. J. Donovan, "Nucleofection mediates high-efficiency stable gene knockdown and transgene expression in human embryonic stem cells," Stem Cells, vol. 26, no. 6, pp. 1436-1443, 2008.

[38] B. Y. Zhou, Z. Ye, G. Chen, Z. P. Gao, Y. A. Zhang, and L. Cheng, "Inducible and reversible transgene expression in human stem cells after efficient and stable gene transfer," Stem Cells, vol. 25, no. 3, pp. 779-789, 2007. 

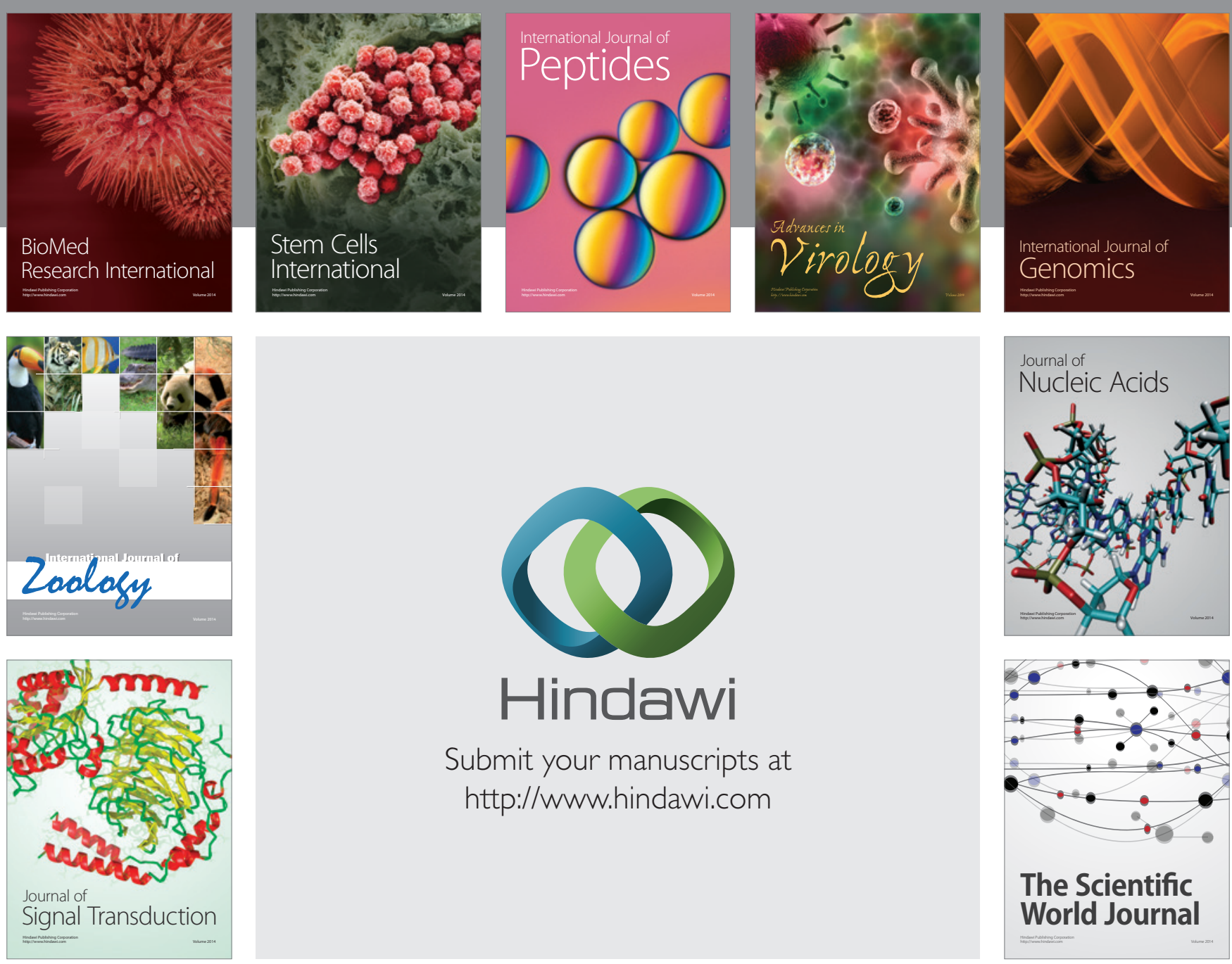

Submit your manuscripts at

http://www.hindawi.com
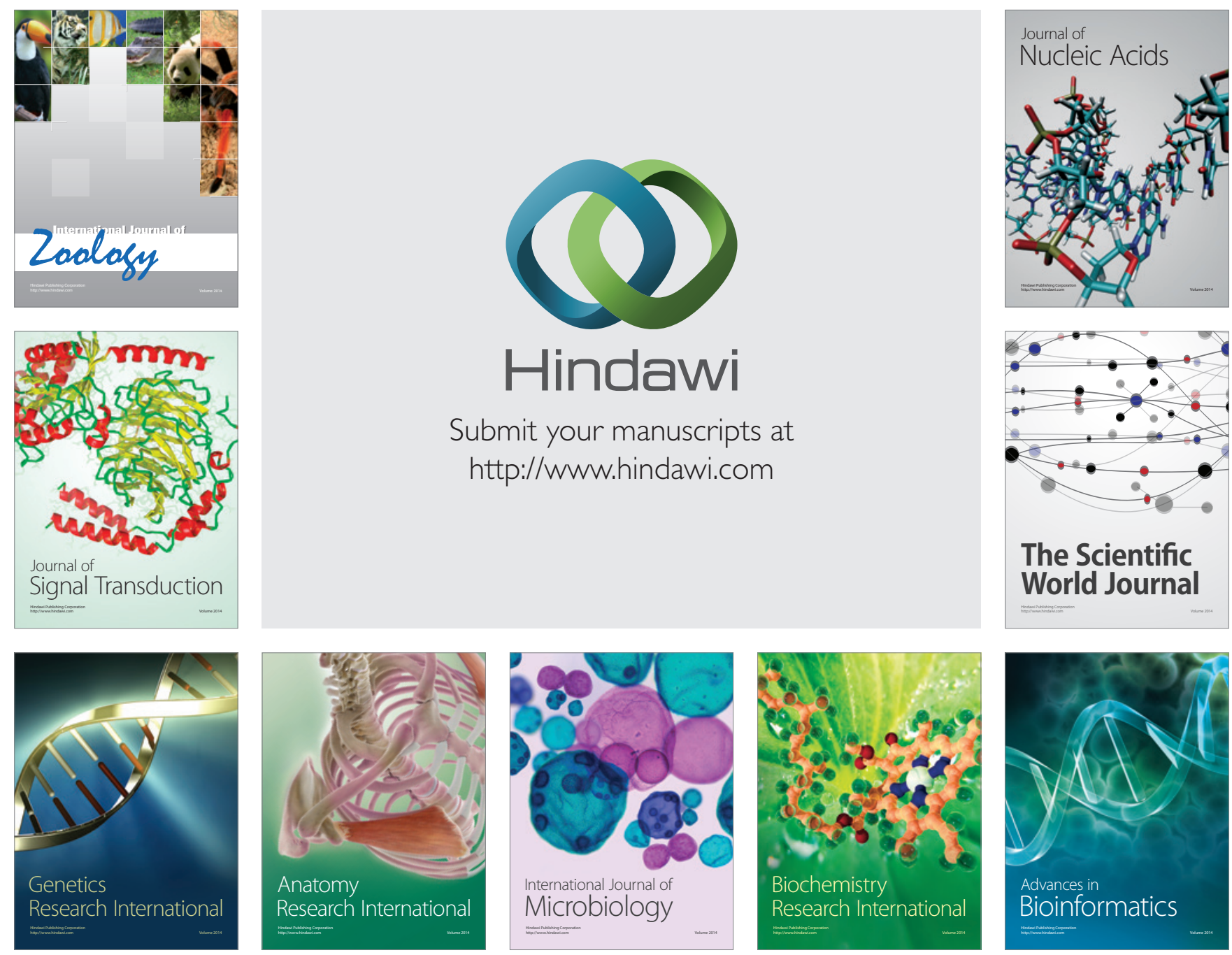

The Scientific World Journal
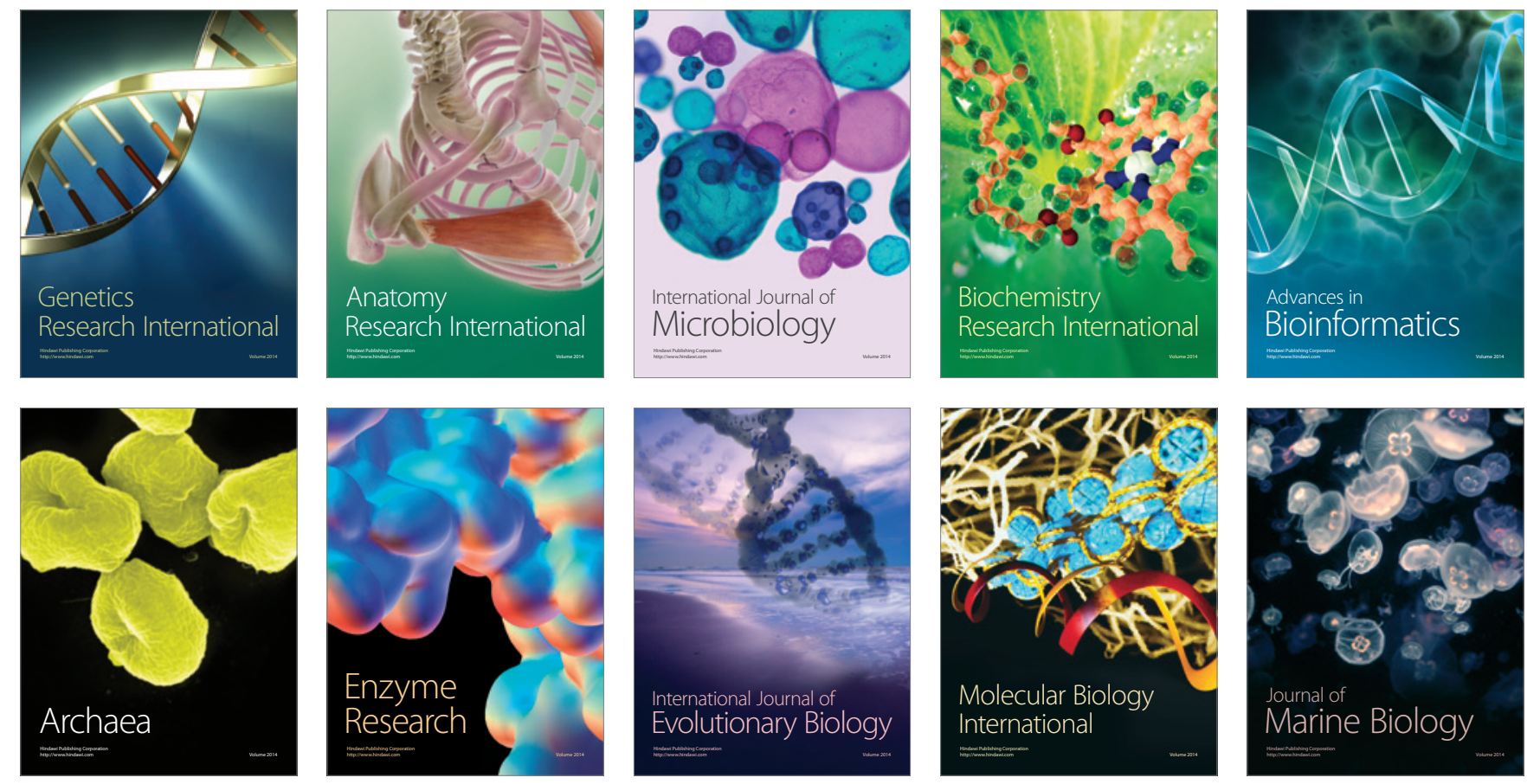